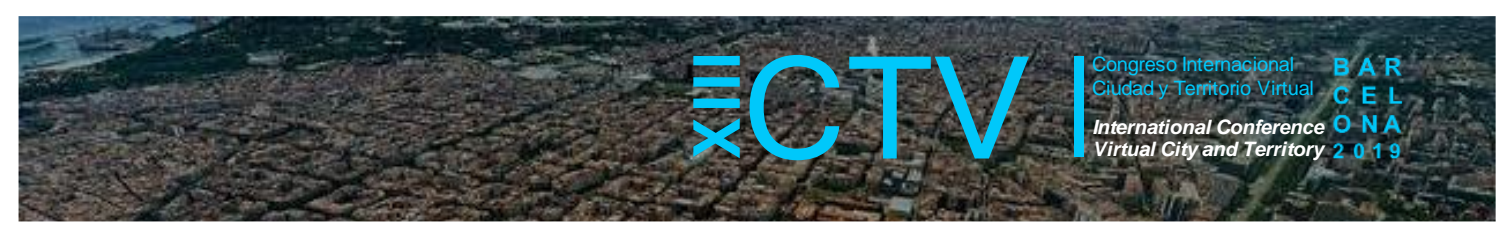

\title{
DE LA NECESIDAD AL PROYECTO: EL DISEÑO DEL ESPACIO PÚBLICO PARA EL CONTROL DEL AGUA Y LA MITIGACIÓN DEL CAMBIO CLIMÁTICO
}

\author{
Mondelli, Francesca Paola ${ }^{\text {; }}$ Rabazo Martín, Marta ${ }^{2}$ \\ Remisión inicial: 2019-06-16; Remisión definitiva: 2019-10-20; Publicación: 2019-12-21
}

Citación: Mondelli, F. y Rabazo Martín, M. (2019). De la necesidad al proyecto: el diseño del espacio público para el control del agua y la mitigación del Cambio Climático. En XIII CTV 2019 Proceedings: XIII International Conference on Virtual City and Territory: "Challenges and paradigms of the contemporary city": UPC, Barcelona, October 2-4, 2019. Barcelona: CPSV, 2019, p. 8634. E-ISSN 2604-6512. DOI http://dx.doi.org/10.5821/ctv.8634

\begin{abstract}
Resumen
El sustituir áreas naturales por superficies pavimentadas impermeables, típicas de la urbanización, no hace más que acrecentar los ya existentes problemas medioambientales de nuestras ciudades. La gravedad de las inundaciones, la falta de recarga de las faldas acuíferas, la erosión de los canales de agua naturales, la contaminación de las corrientes de agua, el aumento de las temperaturas estivas y las islas de calor, la fragmentación de hábitats naturales y la segregación urbana son solo algunas de las consecuencias a las que contribuye.
\end{abstract}

\begin{abstract}
Trabajando correctamente con los espacios públicos urbanos, sus materiales, permeabilidad y distintos niveles de vegetación, y siguiendo algunas pautas de buena praxis, se pueden intentar mitigar todos estos efectos negativos. Un caso claro, en este campo, son las "wáter squares" desarrolladas en el norte de Europa, de las cuales hay importantes ejemplos en Rotterdam, ciudad que se ve fuertemente afectada por su compleja relación con el agua. Estas plazas, planteadas como espacios públicos multifuncionales, tienen la tarea de captar el agua de lluvia y de convertirse, en el caso de lluvias torrenciales o inundaciones, en cuencas de captación que filtran progresivamente el agua al subsuelo, aliviando así el problema del desbordamiento de la red de alcantarillado. Cuando esto no sucede, se quedan vacías y funcionan como áreas relacionales, de juego o de descanso, destacando los beneficios que se derivan de un diseño dinámico del espacio que no sólo está proyectado para una función, sino que es capaz de transformarse en función de las necesidades.
\end{abstract}

Así mismo, según este principio, y en otro orden de importancia en cuanto a su calidad como espacio público pero vital en cuanto a la cantidad de superficie que ocupan en nuestras ciudades, el aparcamiento puede desarrollar un papel fundamental como bien demuestran distintos proyectos y estudios que en las últimas décadas han decidido contribuir a revertir las consecuencias de la urbanización.

Una de las principales consecuencias del cambio climático son las lluvias torrenciales. Los árboles logran reducir los picos de lluvias, deteniendo y evaporando parte del agua, atenuando las lluvias finas y minimizando las fuertes. Las copas de los árboles pueden absorber hasta el $11.1 \%$ de las lluvias anuales, porcentaje que varía con el tipo de árboles usados, el período del año y la duración e intensidad de las lluvias, siendo mayor con árboles de hoja ancha, tormentas estivas de corta duración y baja intensidad.

Es práctica común que en grandes superficies pavimentadas como los aparcamientos se evacue el agua de lluvia tan rápido como sea posible a través del sistema de drenaje, vertiendo el agua en el curso de agua más cercano. Lo que parece ser el uso lógico de estas instalaciones resulta, sin embargo, que cuando las lluvias son muy fuertes, estos canales producen una fuerte descarga, erosionando rápidamente el lecho del arroyo, arrastrando la vegetación y los escombros existentes en sus orillas, y dejando un curso amplio y rocoso. Parece más lógico imaginar un sistema de retención de agua in situ que pueda fomentar una evacuación progresiva del agua, evitando la escorrentía del agua y promoviendo un flujo constante que alimente los acuíferos.

Los estacionamientos también contribuyen al efecto isla de calor producido en las superficies de asfalto que no tienen en cuenta la vegetación, lo que hace que estas superficies sean de $20^{\circ}$ a $40^{\circ}$ más cálidas que las vegetadas, llegando a sufrir una variación de $48,8^{\circ}$ a $17,2^{\circ}$

\footnotetext{
${ }^{1}$ Università degli Studi Roma Tre (Italia), https://orcid.org/0000-0002-2984-128X, francescapaola.mondelli@uniroma3.it; 2 ETSAM, Universidad Politécnica de Madrid (España) y Università degli Studi Roma Tre (Italia), https://orcid.org/00000001-6444-7598, francescapaola.mondelli@uniroma3.it
} 


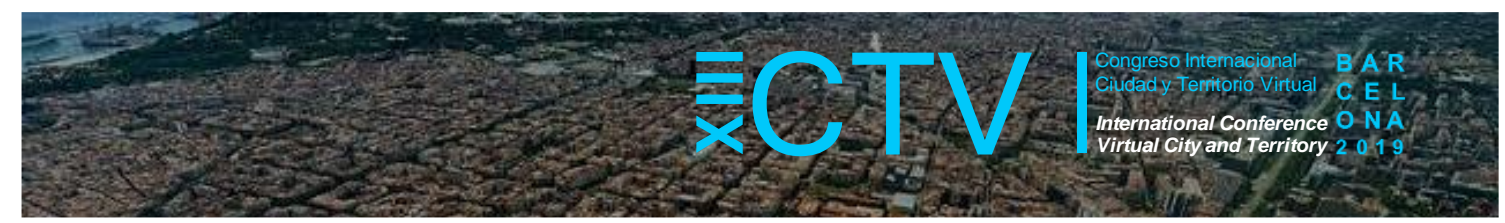

El objetivo de este artículo es remarcar la vital importancia que un correcto diseño de nuestros espacios públicos puede tener sobre la adaptación de nuestras ciudades al cambio climático y a la mitigación de sus efectos. Actuando sobre pequeñas teselas de la superficie urbana con unas bases de buena praxis, podemos crear redes de mayor envergadura que logren minimizar los efectos de una urbanización descontrolada. Imaginemos una nueva red ecológica a la que contribuyan eficazmente todos los espacios públicos, considerando éstos no como actuaciones aisladas sino como un sistema continuo, logrando una mejora considerable de nuestro entorno urbano y trabajando por contrarrestar los efectos del cambio climático.

A través de una metodología inductiva se analizarán distintos casos que en las últimas décadas han trabajado sobre estas temáticas para poder elaborar un decálogo de buenas prácticas.

\section{Abstract}

Substituting natural areas for paved surfaces typical result of urbanization, not only increases the existing environmental problems in our cities. The severity of flooding, lack of groundwater recharging, erosion of natural water channels, increased temperatures and heat islands, the fragmentation of natural habitats, urban segregation, light pollution and increase of car use with the consequence of air pollution are merely some of the consequences to which this contributes.

Designing correctly the urban public spaces, their materials, permeability and different levels of vegetation, and following some good practice guidelines, would help to mitigate all these negative effects. A good example in this field, are the "water squares" developed in northern Europe, mainly in Rotterdam, a city that is strongly affected by its complex relationship with water. These squares, designed as multifunctional public spaces, have the task of capturing rainwater and becoming, in the case of heavy rains or floods, like detention basins that progressively filter the water to the subsoil, alleviating the problem of overflowing the sewer system. When there is no water, they remain empty and function as relational areas or playgrounds, highlighting the benefits of a dynamic design of the space that is not only projected for a single function, but is capable of transforming according to necessity. In another order of importance, maybe secondary in terms of quality as a public space but vital in terms of the amount of surface they occupy in our cities, parking lots can have a main role as well, as different projects and studies in the last decades have pointed out, to contribute to reverse the consequences of urbanization.

One of the main consequences of climate change are heavy rains. The canopy of trees can absorb about $11.1 \%$ of annual rainfall. As a result, this percentage varies focusing on the type of trees used, the time of year and the duration and intensity of rainfall; higher interception rates occur for broadleaf evergreens, warm summer storms, and short, light rain. Tall trees will help to reduce peak rainfalls, with the consequence of stopping and evaporating the water, mitigating light rains and minimizing heavy rains. Therefore, evergreens assist to mitigate surface and water heating.

In addition, a common practice in car parks and large paved surface areas, in general, is to evacuate the rainwater as quickly as possible through the drainage system by pouring that water into a nearby stream. What seems the logical use of these facilities results in a huge amount of water pouring from these canals when the rains are very strong, quickly eroding the stream bed, dragging the vegetation and existing debris of its banks, and leaving a wide rocky watercourse. It is much efficient to produce an on-site water detention system that is able to encourage a progressive evacuation of the water, avoid water runoff and promote a constant influx of water into aquifers. Parking areas also highly contribute to the heat island effect, which happens in asphalt surfaces that do not consider any vegetation, making these surfaces $20^{\circ}$ to $40^{\circ}$ warmer than a vegetated one, reaching a variation of 48.8 degrees to $17.2 \circ$

The aim of this article is to highlight the vital importance that a correct design of our public realm can have on the adaptation of our cities to climate change and the mitigation of its effects. Acting on small areas of the urban surface with good practice bases, we can create larger networks that minimize the effects of an uncontrolled urbanization. We should imagine a new ecological network to which all public spaces contribute effectively to, considering these not as isolated actions but as a continuous system, achieving a considerable improvement of our urban environment and working to counteract the effects of climate change.

Through an inductive methodology, we will analyze different cases that in recent decades have worked on these issues in order to develop a new code of good practices.

Palabras Clave: nuevas ecologías urbanas; aparcamientos sostenibles; teselas urbanas; espacio público activo

Key words: new urban ecologies; sustainable parking lots; urban tiles; active public space 


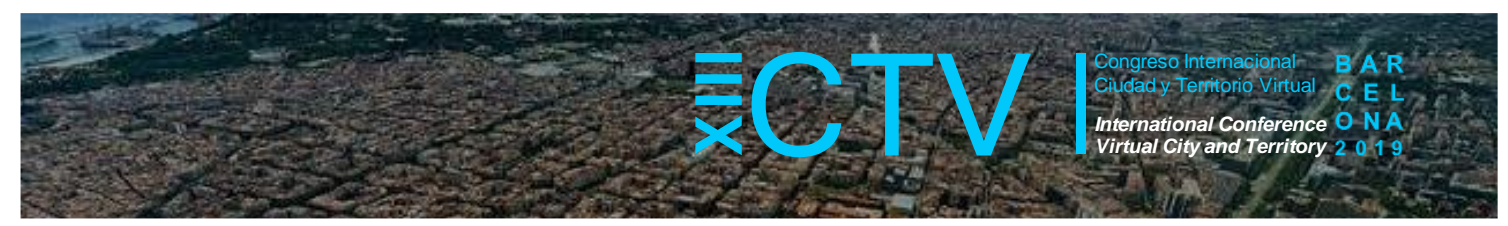

\section{El cambio climático y el proyecto de paisajismo}

Si la arquitectura del paisaje pretende ser un instrumento de intermediación entre las necesidades humanas y el medio ambiente, en nuestra época, tan fuertemente caracterizada por el fenómeno del cambio climático, el arquitecto paisajista no podrá hacerlo sin enfrentarse a los problemas y las transformaciones. derivados de dicho cambio. El concepto mismo de paisaje incluye la intervención humana y, por lo tanto, las llamadas obras de asentamiento humano que, de forma ligera 0 más consistentemente, dan lugar con el tiempo a modificaciones sustanciales del entorno natural. Sin embargo, el paso de una condición más natural y no contaminada a una más artificial y alterada no debe verse con un significado necesariamente negativo, sino como la consecuencia inevitable del desarrollo de la vida humana en el medio ambiente. Paisaje y territorio son dos términos que indican, de diferentes maneras, el ambiente antropizado: el primero que sigue una connotación con un valor más estético y cultural; el segundo, como nos enseña Eugenio Turri, nos muestra la extensión física en que se atestiguan las transformaciones humanas.

Así hasta el momento en que la relación entre el hombre y el medio ambiente estaba en equilibrio, las obras de antropización han dado forma al territorio, transmitiendo la cultura del pasado, desarrollada en una convivencia armoniosa con la naturaleza; con el progreso tecnológico de la era moderna, a partir de la segunda revolución industrial, las acciones antrópicas han experimentado una aceleración tal que rompe el equilibrio preexistente causando fuertes cambios negativos para el medio ambiente (Mattogno ${ }^{2}$ ). No es casualidad que solo recientemente se haya introducido el concepto de paisaje, asociado con mayor frecuencia al tema de la "protección": es en el momento en que se produce la separación del hombre de la naturaleza, rompiendo la relación armónica, que nos hemos preocupado de recuperar esta relación a través de un enfoque de "salvaguardia" y "protección" también determinado por la conciencia de las alarmantes consecuencias introducidas por la ruptura de este equilibrio.

El cambio climático es probablemente la más importante de estas consecuencias. Aunque debe tenerse en cuenta que este aspecto también debe considerarse, en parte, como una transformación natural de las características ambientales a lo largo de milenios, también es cierto que este fenómeno hoy es principalmente provocado y, sin duda, acelerado por acciones antrópicas demasiado violentas y rápidas, tanto de no dejar tiempo suficiente para que el medio ambiente se adapte progresivamente a dichos cambios. Podríamos definir el cambio climático como un "efecto secundario" de las actividades humanas (Di Paola, Pellegrino ${ }^{3}$ ), que corre el riesgo de modificar irreparablemente el planeta y las características que han hecho posible que la vida se desarrolle hasta ahora. Pero si es a partir de un equilibrio perdido entre el hombre y la naturaleza que se ha generado este escenario, será precisamente a partir de este punto que tendremos que comenzar de nuevo para responder a los problemas ambientales contemporáneos. Esto significa el abandono gradual de un enfoque de ingeniería técnica para resolver los problemas generados por el cambio climático (como inundaciones, sequías, sobrecalentamiento de las ciudades), redescubriendo en la naturaleza los recursos para mitigar tales efectos. Esta es la razón por la cual hoy es necesario plantear el proyecto de paisaje como respuesta al cambio climático: a diferentes escalas, desde acciones puntuales de micro-

\footnotetext{
${ }^{2}$ Mattogno, C. (2014) "Ambiente" in Ventuno parole per l'urbanistica. Roma: ARACNE editrice.

${ }^{3}$ Di Paola, M. Pellegrino, G. (2018) Nell'Antropocene. Etica e politica alla fine di un mondo. Roma: Derive Approdi
} 


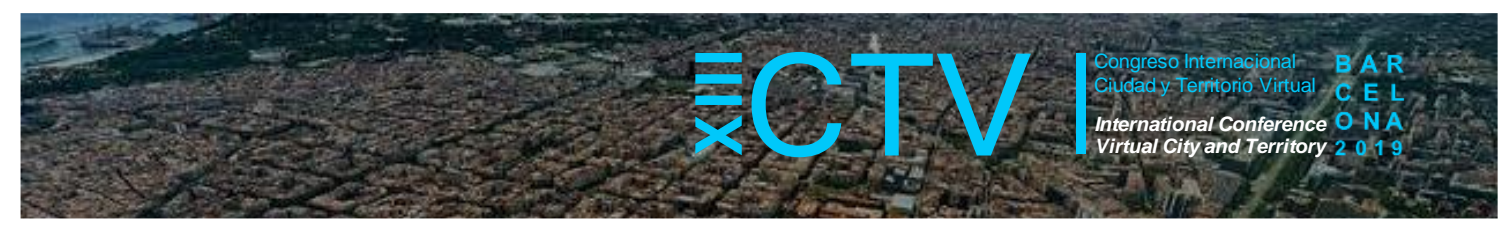

paisajes dentro de los centros urbanos, hasta la planificación del paisaje a mayor escala, el objetivo es proyectar con la finalidad de la adaptación climática. Este es el nuevo enfoque que se está introduciendo en muchos países del mundo para desalentar las obras artificiales duras y con poca capacidad de adaptación, proponiendo un diseño más suave y flexible capaz de adaptarse a la dinámica natural de los fenómenos ambientales, combinando la lucha contra el cambio climático con la regeneración urbana. Proyectos que sean, por lo tanto, capaces de brindar oportunidades de mejora en nuestras ciudades, no solo desde un punto de vista técnico y de contención de riesgos, sino como un punto de partida para la creación de espacios de reunión, áreas públicas que cumplen simultáneamente la doble función ambiental y social. Es también por estas razones, que la ciudad es el campo de aplicación más adecuado para la experimentación de un proyecto de paisaje que tiene en cuenta los desafíos climáticos; es aquí donde prevalece lo artificial sobre lo natural y, por lo tanto, el famoso equilibrio se ve más comprometido.

En las ciudades contemporáneas, el desafío es transformar los riesgos en oportunidades, introduciendo el concepto de resiliencia, o la capacidad de las ciudades para adaptarse a los cambios repentinos en el contexto ambiental en el que surgen. Aunque la arquitectura en sí surge de la necesidad humana de adaptarse a las condiciones ambientales $\mathrm{y}$, por lo tanto, principalmente a los diferentes climas en los que se desarrolla la vida y la sociedad, nos hace pensar cómo, con el avance de la tecnología, se consideró posible descuidar el problema climático, resolviéndolo con recursos tecnológicos que solo han empeorado las consecuencias, lo que hace necesario hoy volver a integrar este tema en el debate urbano, paisajístico y arquitectónico. Poniendo el clima nuevamente en el centro de la arquitectura y las políticas urbanas, nos preguntamos cuáles son los cambios resultantes en términos de diseño. ¿Cómo cambia el enfoque paisajista con referencia al cambio climático? ¿Cómo puede intervenir el proyecto de paisaje en la ciudad para mitigar sus efectos?

\section{Cambia el clima, cambia el proyecto de paisajismo}

Las respuestas a estas preguntas son muchas y pueden dividirse en varios niveles: por un lado, hay consideraciones de una naturaleza más teórica y general; por otro lado, existen aspectos prácticos reales que pueden y deben implementarse cada vez que se aborda el proyecto, ya se refiera éste la unidad de habitativa o a la intervención en espacios abiertos y el paisaje a escala territorial. La primera respuesta viene determinada por el cambio metodológico del diseño que reemplaza la estaticidad de la ciudad con su dinamismo intrínseco, más visible actualmente debido a la velocidad de las transformaciones. No se trata tanto de contrastar los cambios, sino de adquirir la capacidad y la ductilidad de vivir con ellos. El proyecto, concebido de manera flexible, ya no tiene una estructura rígida, sino una estructura abierta, dispuesta a cambiar con el tiempo (Manigrasso ${ }^{4}$ ). Considerando el evento catastrófico como la excepción en un estado de regularidad climática, la planificación urbana debe enfocarse en el uso del espacio público en el mayor tiempo en que no se ve afectado por el fenómeno, concibiendo así lugares utilizables que puedan servir para mitigar los riesgos cuando ocurre la emergencia.

\footnotetext{
${ }^{4}$ Manigrasso, N. (2012). Verso la Città ad_Attiva. Rispondere ai cambiamenti climatici attraverso una nuova concezione del tempo nei processi e negli esiti progettuali. En: Atti della XV Conferenza Nazionale SIU - L'urbanistica che cambia. Rischi e Valori. Pescara, Italia.
} 


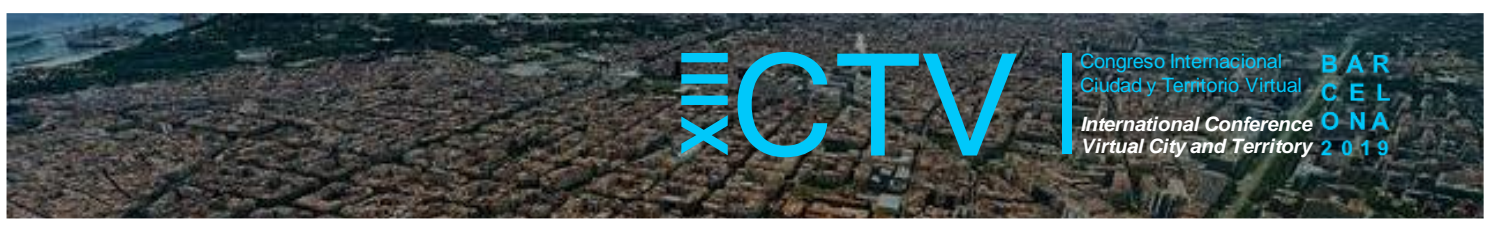

Un segundo punto se refiere a la transición desde una visión puramente estética del paisaje a la afirmación de su papel como "sistema inmunitario" de la ciudad (Celestini ${ }^{5}$ ). Es a travésdel verde, de hecho, que se intenta conseguir una mejor gestión de los recursos hídricos, contrarrestar la contaminación del aire y frenar el sobrecalentamiento y las conocidas islas de calor que se crean en nuestras ciudades. Como dice Andreas Kipar ${ }^{6}$, fundador del estudio de arquitectura del paisaje LAND, con sede en Milán, "necesitamos que la naturaleza trabaje para nosotros", estimular y llevar a cabo un "cuidado del verde" capaz de sanar los grandes centros urbanos cada vez más afectados por la migración de habitantes desde la provincia y el campo a la gran ciudad. Es precisamente este escenario que mantiene la atención puesta sobre la cantidad de espacios verdes presentes en la metrópoli de hoy en día, es decir, donde el equilibrio entre la naturaleza y las obras antrópicas está más en desequilibrio.

A tal propósito, el investigador Phillipp Gärtner ha creado imágenes satelitales que resaltan el porcentaje de verde en las principales capitales europeas, comparando primero las que superan los dos millones de habitantes, y luego las costeras, separadas de las demás por la presencia de una gran proporción de tierra no cultivable. La investigación es muy interesante mostrando las ciudades europeas más verdes, pero sobre todo aquellas "no tan verdes", destacando las deficiencias incluso en ciudades que parecen muy ricas en espacios naturales, como Roma y Milán.

Figura 1. Greenest European metrópolis

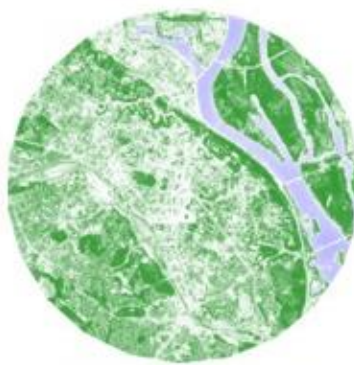

Kiev / Ukraine (NDVI 0.389)

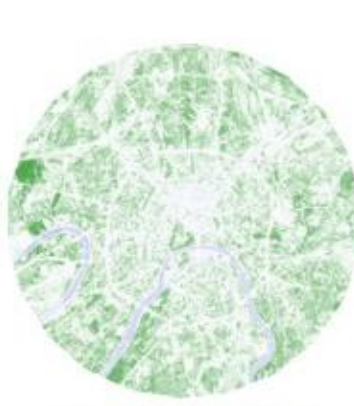

Moscow / Russia (NDVI

$0.143)$

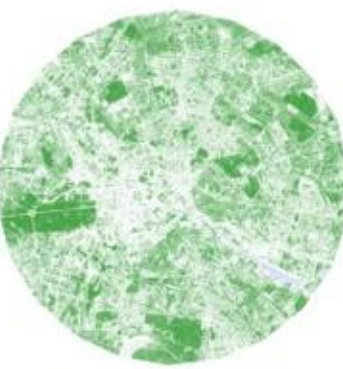

Berlin / Germany (NDVI 0.246)

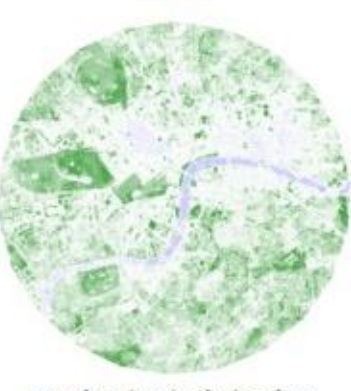

London / United Kingdom

(NDVI 0.135)

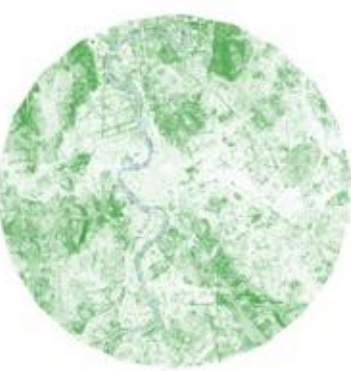

Rome / Italy (NDVI 0.17)

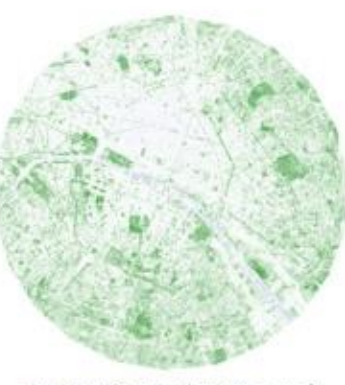

Paris / France (NDVI 0.119)

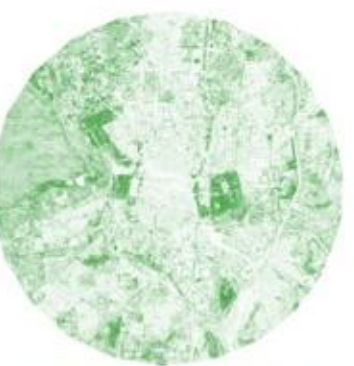

Madrid/Spain (NDVI 0.154)

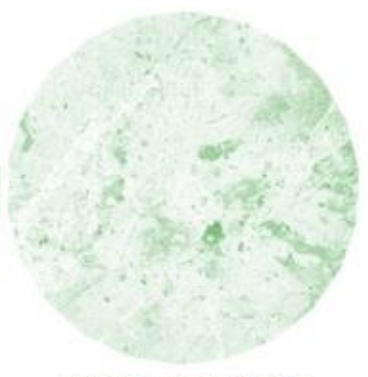

Athens / Greece (NDVI

0.088)

Nota: Ciudades con más de 2 millones de habitantes: Kiev, Berlín, Roma, Madrid, Moscú, Londres, París, Atenas. Fuente: Philippe Gartner.

\footnotetext{
${ }^{5}$ Celestini, G. intervención en el artículo:Di Gregorio, R. (2019). Il progetto di paesaggio come risposta alle sfide climatiche. Recuperado de https://www.edilportale.com/news/2019/05/ambiente/il-progetto-di-paesaggio-come-rispostaalle-sfide-climatiche 70277 52.html

${ }^{6}$ Kipar, A. intervención en el artículo: Traldi, L. (2018). Design e Climate Change. L'architettura del paesaggio che salva la vita. In Dcasa, novembre 2018, pp. 56-60.
} 


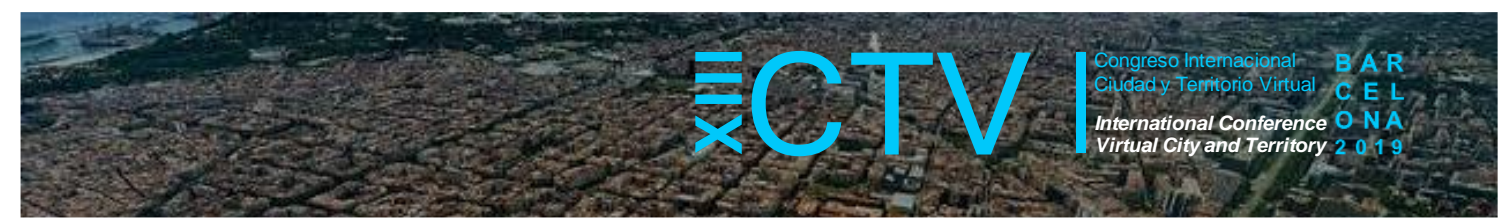

Sin embargo, la necesidad que sentimos hoy de querer "salvar la naturaleza" debe enriquecerse con la conciencia de que "la naturaleza puede salvar la ciudad" (Celestini ${ }^{7}$ ). Este es un punto clave del discurso, porque es aquí donde entendemos la importancia para el paisajista de redescubrir en la naturaleza los elementos, las técnicas y los equilibrios fundamentales del proyecto, a fin de restaurar esa armonía típica de la arquitectura tradicional. Justo en el punto de encuentro entre la necesidad de proteger el medio ambiente y la capacidad del medio ambiente para mejorar la calidad de nuestras ciudades, encontramos el proyecto de paisaje contemporáneo, que tiene como objetivo dar nuevas respuestas en la lucha contra el cambio climático. Sobre este tema, es fundamental la lección de Pietro Laureano, quien durante años ha estado promoviendo el conocimiento tradicional como un camino para combatir la desertificación, en defensa de los ecosistemas en peligro.

¿Cómo puede, a un nivel más práctico, la arquitectura del paisaje intervenir influyendo y contrarrestando los efectos de estos cambios, especialmente en las ciudades? Afortunadamente, hay muchos ejemplos, repartidos por todo el mundo, de buenas prácticas tanto a nivel de políticas urbanas como a nivel de proyectos individuales de espacios abiertos. Ciudades como Nueva York, Copenhague y Singapur son hoy un modelo por el rol central que han otorgado a la lucha contra el cambio climático y sus consecuencias, especialmente en el campo de la gestión del agua, dentro de su planificación urbana.

\section{Experiencias, estrategias y buenas prácticas de proyecto}

El espacio abierto es el lugar de las continuas transformaciones y lo es, especialmente, en los lugares más cercanos a la naturaleza, como las costas o cursos fluviales que atraviesan los centros urbanos. Lugares complejos, donde la tensión entre las construcciones antrópicas y el entorno natural es tan fuerte que hace que la visión del paisaje sea un "sistema" que debe ser tratado, analizado y diseñado en su complejidad y su constante mutabilidad.

Para encontrar buenos ejemplos de proyectación, debemos referirnos a aquellas realidades que debido a sus características morfológicas y climáticas siempre han tenido que enfrentarse a la necesidad de desarrollarse en un contexto ambiental más difícil. Singapur ciertamente se sitúa dentro de estos casos. La isla se encuentra en una condición donde los recursos hídricos son prácticamente ausentes: esto ha significado que el gobierno inicialmente estableció acuerdos con Malasia para el suministro de agua, para posteriormente decidirrse a invertir en un imponente programa de gestión, recolección y reciclaje del agua, llamado ABC Water Active, beautiful and clean wáter programme (programa de aguas activas, hermosas y limpias). "Cualquier otra política debe arrodillarse ante la importancia de la conservación del agua", son las palabras de Lee KuanYew, primer ministro hasta 1990, y de hecho las características físicas y climáticas de Singapur han hecho necesario construir infraestructuras para el drenaje durante los monzones, canales y embalses para aliviar los efectos de las inundaciones frecuentes. Desde la década de 1980, el gobierno de Singapur ha decidido experimentar con una integración entre estas estructuras de control del agua y el desarrollo urbano: una inversión de la tendencia que, en lugar de distanciar a las personas del agua, elige acercarse a ella, transformando canales y cuencas en espacios verdes y azules que la población puede disfrutar de forma recreativa.

\footnotetext{
${ }^{7}$ Celestini, G. intervención en el artículo Di Gregorio, R. (2019). Il progetto di paesaggio come risposta alle sfide climatiche. Recuperado de https://www.edilportale.com/news/2019/05/ambiente/il-progetto-di-paesaggio-come-rispostaalle-sfide-climatiche $70277 \frac{1}{52 . h t m l}$
} 


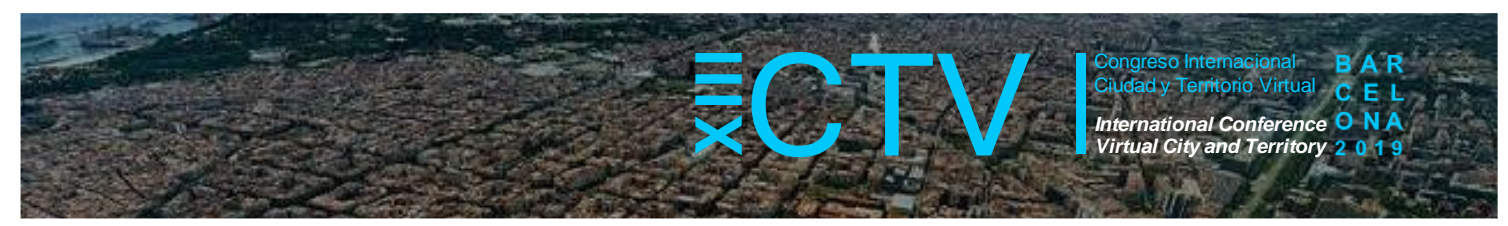

En 2006, la introducción del $A B C$ Water Programme demuestra los efectos positivos de la introducción del agua en el espacio urbano, mejorando el paisaje y contribuyendo al mismo tiempo a la gestión de las inundaciones. No más infraestructuras de hormigón lejos de la ciudad, sino pintorescos paisajes de agua que se integran en el contexto urbano. El programa se inspira explícitamente en el ejemplo canadiense Melbourne Water, que en Australia lanzó el proyecto "Diseño urbano sensible al agua", que muestra la importancia del recurso hídrico dentro de los centros urbanos. El agua, de hecho, además de mejorar la estética del paisaje urbano, ayuda a contrarrestar los efectos del cambio climático, mejorando el microclima y reduciendo las islas de calor. Esta es una investigación que destaca la importancia de una gestión estratégica de un elemento como el agua, especialmente en referencia a los fenómenos de sequía y las inundaciones cada vez más frecuentes, y que ha tenido tanto éxito que se ha vuelto a proponer también en Gran Bretaña, en una versión adaptada al territorio inglés llamada "Diseño urbano sensible al agua en el Reino Unido".

En el contexto europeo, los países históricamente caracterizados por mantener una relación compleja con la gestión del agua han puesto en marcha planes y proyectos destinados a abordar las transformaciones derivadas del cambio climático, en particular los Países Bajos y Dinamarca. En Copenhague, el Plan de Adaptación al Clima coloca a la ciudad frente a los desafíos de un clima cada vez más difícil de controlar. La ciudad, sujeta a eventos de lluvia extrema, ha llevado a cabo estudios para proyectar el impacto de las inundaciones debido al cambio climático en el futuro, con el fin de identificar las áreas más vulnerables. El riesgo de inundación se aborda mediante intervenciones que se adaptan a un posible estado de emergencia, permitiendo que el exceso de agua sea drenado o recogido a través destorm wáter roads, canales que transportan el agua hacia los lagos y el puerto; detention roads para almacenamiento de agua; detention areas para la recolección de grandes cantidades de agua, como parques que se pueden transformar en lagos si es necesario; Green roads para canalizar el agua hacia caminos secundarios (Bassolino ${ }^{8}$ ). El objetivo no es centrarse solo en contener los riesgos, sino en aprovechar la oportunidad de mejorar la calidad de los espacios de la ciudad, creando áreas recreativas más ecológicas y sostenibles, para el disfrute de parte de los ciudadanos. Dentro de las guías del plan, de hecho, se desarrollan proyectos que combinan la gestión de riesgos con la naturalización de la ciudad, la introducción de rain gardens y suelos permeables, junto con medidas destinadas a reducir el tráfico y, por lo tanto, las emisiones de gases contaminantes, dando impulso a la creación de ciclovías que a su vez actúan como canales para recoger agua en casos de inundación.

El mismo enfoque adaptativo, pero a una escala más territorial, es el elegido por el programa holandés "Room for the Rivers", un plan de control de inundaciones que consiste en crear más espacio para los cursos fluviales, aumentando la profundidad de los cursos, creando canalizaciones y eliminando las estructuras de contención que no dejan espacio para el agua, a fin de restaurar los paisajes a lo largo de los ríos que pueden filtrar y absorber naturalmente el agua en caso de inundaciones.

Pero incluso a escala urbana, los Países Bajos se han ocupado de la gestión del agua a través de proyectos de espacios públicos flexibles y dinámicos. Este es el caso de la ciudad de

\footnotetext{
${ }^{8}$ Bassolino, E. (2019). The impact of climate change on local water management strategies. Learning from Rotterdam and Copenhagen. In UPLanD, n4, pp. 21-40.
} 


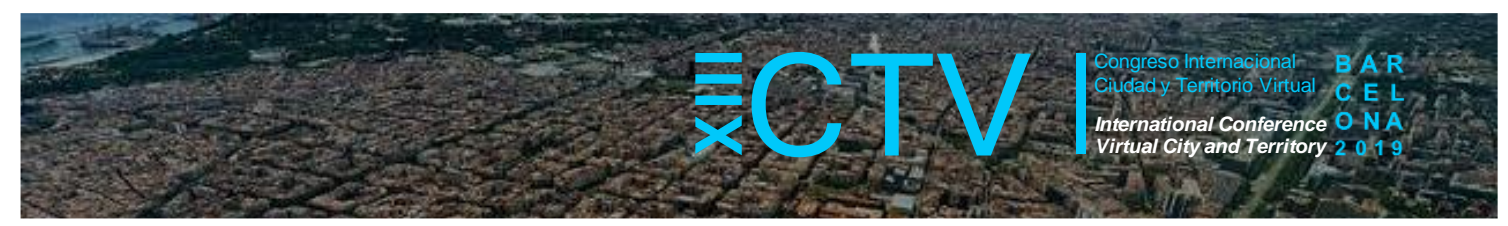

Rotterdam, que, con su Climate Change Adaptation Strategy, pretende ser totalmente resiliente al cambio climático para 2025. Las intervenciones planificadas se distribuyen por toda la ciudad y prefieren un enfoque de impacto leve, mediante el uso de techos verdes, suelos permeables y los famosos Water Squares, desarrollados por los diseñadores De Urbanisten que crearon el "Water Square Benthemplein" en el distrito de Zoho, un sector urbano que se ha utilizado como un verdadero laboratorio para experimentar con nuevas técnicas para la adaptación climática de bajo impacto.

Figura 2. Master plan diseñado por BIG Team para la recuperación de las orillas del río en Lower Manhattan, "Big U-Rebuild by design” (izquierda) / "Water Square Benthemplein" desarrollados por los diseñadores De Urbanisten, en Rotterdam (derecha)
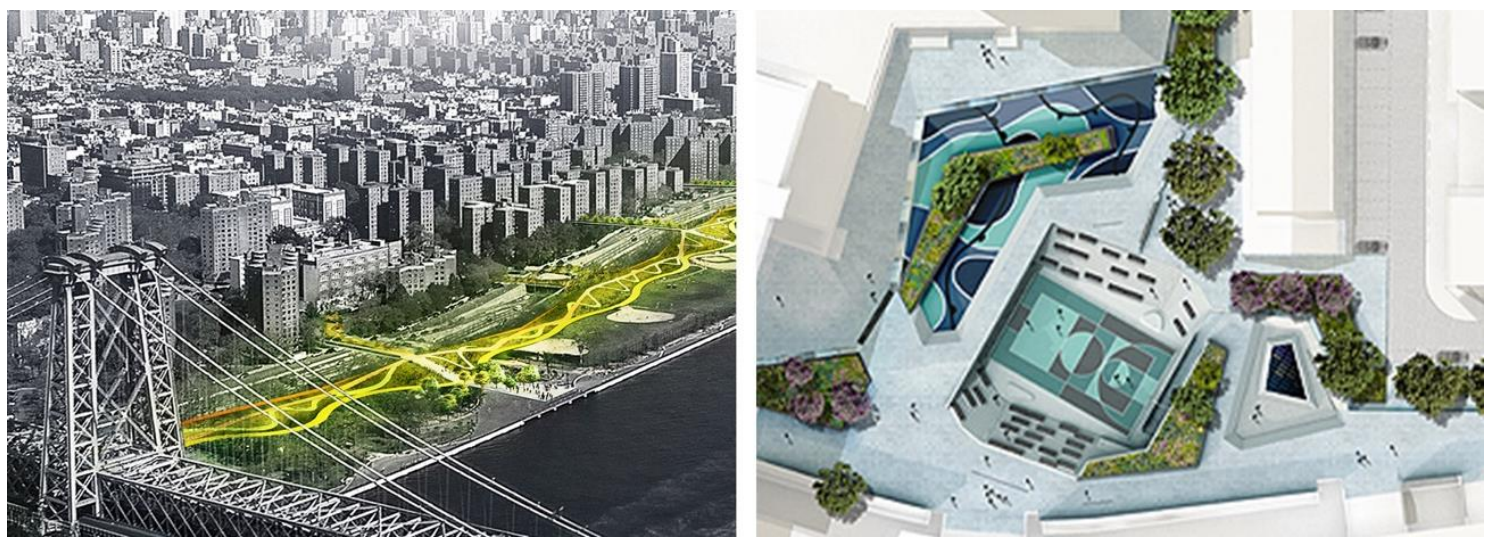

Al otro lado del océano, en Manhattan, las condiciones climáticas se intensificaron hasta el punto de causar catástrofes como la del huracán Sandy en 2012. Como resultado, la ciudad de Nueva York decidió equiparse con un Masterplan diseñado por BIG Team para la recuperación de las orillas del río en Lower Manhattan, "Big U-Rebuild by design". La intervención, aunque surge de una necesidad inicial de adaptar las riberas a eventos climáticos excepcionales, al mismo tiempo quiere responder a la demanda de la ciudad de una remodelación del área.

El proyecto, que se desarrollará a lo largo de los años, adopta un enfoque a medio camino entre el técnico y el naturalista, proponiendo un área verde que puede inundarse en caso necesario, pero que puede separarse del resto de la ciudad gracias a un sistema de estructuras móviles. Estos elementos se integran en el diseño urbano, tanto como decoración como parte de los equipamientos multifuncionales incluidos en el proyecto.

Son numerosos los casos de proyectos de paisajismo que han comenzado hábilmente a trabajar en un diseño estratégico como respuesta al cambio climático. En Asia, el estudio Turenscape ha estado trabajando en esta dirección durante años: un ejemplo es el Parque Natural Yanweizhou en la ciudad de Jinhua, un proyecto para un área fluvial que propone una gestión de las inundaciones puramente ecológica, gracias a una terraza verde cubierta con vegetación nativa adecuada para soportar las crecidas; o incluso el Parque Houtan en Shanghai, construido en una antigua área industrial a lo largo del río Huangpu, que también tiene como objetivo un control ecológico de los flujos de agua, inundaciones y el tratamiento de la contaminación del agua. 


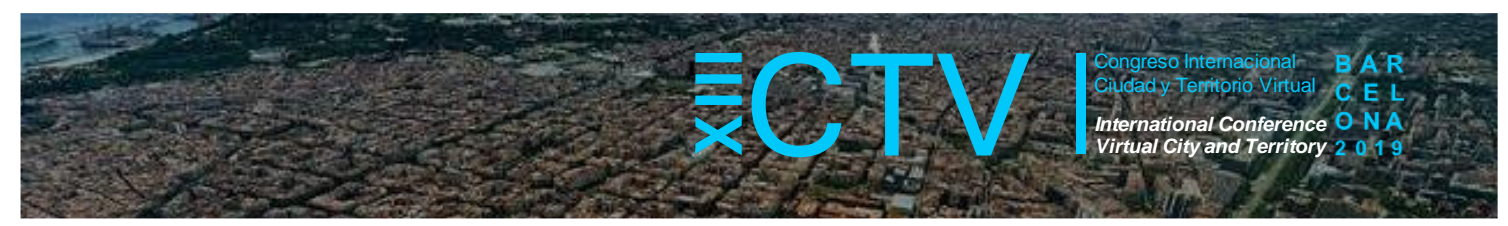

\section{Hacia una ciudad permeable}

El sustituir áreas naturales, bien sean estas praderas, arbustivas o arboledas, por superficies pavimentadas típicas de la urbanización, no hace más que acrecentar los ya existentes problemas medioambientales de nuestras ciudades. La gravedad de las inundaciones, la falta de recarga de las faldas acuíferas, la erosión de los canales de agua naturales, la contaminación de los cursos de agua, el aumento de las temperaturas estivas y las islas de calor, la fragmentación de hábitats naturales, la segregación urbana, la contaminación lumínica y el aumento del uso del coche, con la consecuente mayor contaminación del aire, son solo algunos de las consecuencias a las que contribuye.

En zonas sin urbanizar, los bosques, humedales y otras áreas verdes están conectadas con las infraestructuras verdes regionales, grandes sistemas de vegetación que atraviesan el territorio, importantes de preservar por su capacidad para purificar el aire, absorber las grandes lluvias y proteger los hábitats animales.

En las zonas urbanizadas y, de especial modo, en las urbes, estas zonas verdes permeables han sido masivamente sustituidas por asfalto y cemento. Tanto es así que ciudad y asfalto han llegado a ser conceptos indisolubles quedando ligados en el imaginario de los ciudadanos. No se pretende hacer un alegato contra el asfalto, material que ha contribuido muy positivamente al desarrollo urbano por sus características higiénicas (no produce polvo con el paso de los vehículos), atenúa el ruido de los coches y su coste de instalación y manutención es bajo. Si intentaremos descifrar las pautas de buena praxis que pueden ayudar a mitigar sus efectos negativos derivados de un uso abusivo.

Dentro del ámbito urbano existe una tipología de espacio como es el aparcamiento en superficie que funciona como estandarte del uso desmesurado del asfalto. Aparcamiento significa para la mayor parte de nosotros una gran superficie de asfalto con líneas blancas que, en el mejor de los casos, cuenta con algún árbol o pérgola. Abandonado y olvidado por los encargados de diseñar nuestro entorno urbano, han quedado así fuera de los ámbitos que consideramos públicos, reduciéndose a un espacio desagradable y marginal. Proponemos así el aparcamiento como un espacio desde donde poner en práctica una nueva conciencia sobre la gestión del agua y de la permeabilidad del terreno.

Con respecto a la gestión del agua uno de los temas más importantes reside en el agua de escorrentía. Muchos estudios apoyan la importancia de usar materiales permeables para poder reducir el agua de escorrentía y permitir que se pueda infiltrar y recargar correctamente los acuíferos. La escorrentía de 400m2 de una superficie pavimentada de un aparcamiento es 16 veces mayor que un área vegetada de similares dimensiones. En una zona arbolada, el 10\% del agua de lluvia es de escorrentía, el $25 \%$ se infiltra en el subsuelo, otro $25 \%$ se encauza y se une a los cursos de agua existentes y el $40 \%$ se evapora; en un entorno urbanizado con el 75 $100 \%$ de superficies impermeables, se produce más del $55 \%$ de escorrentía. Y no sólo eso, el calor acumulado, en los materiales impermeables, calienta el agua de lluvia que los atraviesa disminuyendo los niveles de oxígeno de la misma (Mayer, 2005).

El introducir pequeños espacios verdes en los aparcamientos ayuda a crear puntos donde el agua de lluvia pueda ser absorbida. Al mismo tiempo, incluir árboles en su diseño estos pueden absorber las lluvias finas y parte de las medias, ayudando a temporizar la evacuación del agua 


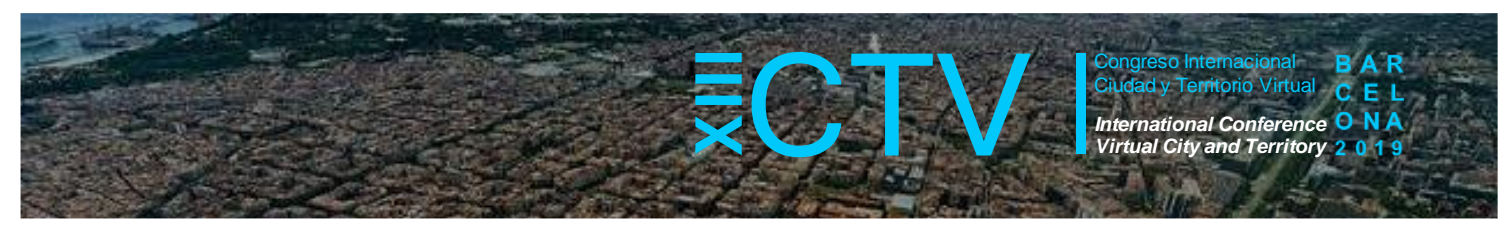

y mejorando el sistema general (Childs, 1999)

Hay tres consideraciones importantes a la hora de diseñar un aparcamiento desde el punto de vista del comportamiento hídrico:

- Los espacios verdes dedicados a poder recoger el agua deben de estar rehundidos con respecto a la cota del aparcamiento para que el agua realmente pueda acceder a ellos.

- Las plantaciones de altura (árboles) logran reducir los picos de lluvias, deteniendo y evaporando el agua, atenuando las lluvias finas y minimizando las fuertes. Los árboles de hoja perenne ayudan a mitigar el calentamiento de las superficies y del agua. Los caducifolios sin embargo conviene que no estén cerca de las lagunas (de retención o Smart ponds) para que no colapsen los filtro con sus hojas. En un estudio en Sacramento, California, se constató que las copas de los árboles pueden absorber alrededor del $11.1 \%$ anual. Este porcentaje varía con el tipo de árboles usados, el período del año y la duración e intensidad de las lluvias, siendo mayor con árboles perennes de hoja ancha, tormentas estivas de corta duración y baja intensidad. Así el mismo estudio calculó una intercepción del $36 \%$ para los bosques urbanos perennes de hoja ancha y coníferas durante el verano en Sacramento y solo un $2.4 \%$ de intercepción en bosques urbanos caducos de hoja ancha y de coníferas de tamaño medio durante el invierno en una tormenta fuerte (200 años) (Childs, 1999)

- Las tuberías de drenaje es estos espacios deberían estar perforadas (y cubiertas con una fibra anti-raices) para poder permitir que el agua se filtre y alcance los acuíferos por debajo de las superficies impermeables (si estas no se pueden evitar)

Aunque no toda el agua proveniente de la lluvia de una zona urbanizada está contaminada, si lo está la proveniente de aparcamientos y calles debido a los metales, aceites y demás elementos contaminantes precedentes de los coches (donde además se calienta reduciendo su contenido en oxígeno), que son lavados y transportados por las lluvias hasta los acuíferos, contaminando así las faldas y destruyendo ecosistemas y hábitats animales. Es por esta razón que el agua de lluvia que corre por otro tipo de superficies (edificios, tejados, ...) no debe evacuarse a través de superficies destinadas a coches, si no dirigirse a zonas donde pueda ser filtrado y filtrase en el terreno. Al mismo tiempo, el agua que no es recolectada y que no llega a las faldas acuíferas, no puede en ningún modo re usarse. Estas superficies no consumen agua potable pero no contribuyen tampoco a la percolación del agua de lluvia.

Estudios realizados señalan que el agua que se pierde por no ser recolectada en las superficies impermeables varía de los 54.5 billones de litros en Dallas hasta los 502.7 billones de litros en Atlanta. Esto se traduce en que Atlanta en 1997 "perdía" la cantidad de agua para abastecer las necesidades domesticas diarias de 1.5 millones a 3.6 millones de personas al año (Ben-Joseph, 2012).

Otro de los daños provocados por el agua de escorrentía es la desestabilización de los cursos naturales de agua. Una práctica común en los aparcamientos y grandes superficies pavimentadas es evacuar el agua de lluvia lo más rápido posible a través del sistema de drenaje, vertiendo esa agua en el arroyo más cercano. Lo que parece el uso lógico de estas instalaciones da como resultado, sin embargo, que cuando las lluvias son muy fuertes hay una gran cantidad de agua saliendo de estos canales, el lecho del arroyo se erosiona rápidamente, arrastrando la vegetación y los escombros existentes de sus riberas, y dejando un amplio curso de agua rocosa. Parece mucho más lógico imaginar un sistema de retención del agua en el 


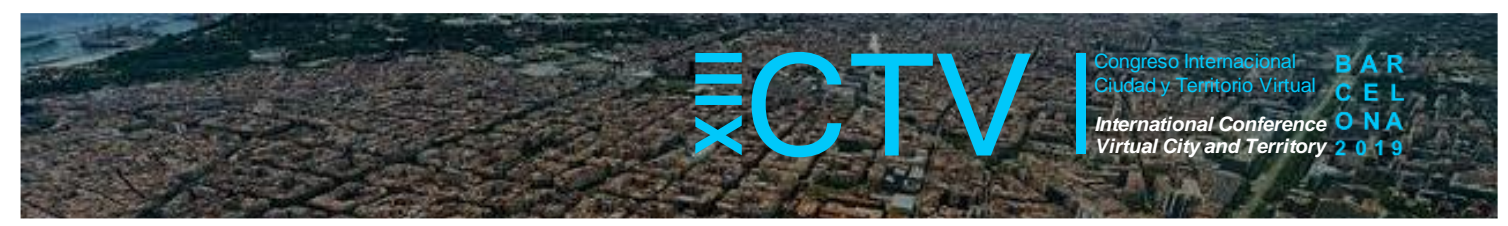

sitio que pueda alentar una evacuación progresiva del agua, evitando la escorrentía de agua y promoviendo una afluencia constante de agua en los acuíferos. Estos estanques de retención deben ir acompañados de vegetación apropiada (como rain gardens) para que no aparezcan como simples cuencas vacías cuando no hay agua.

Los efectos negativos de las grandes superficies asfaltadas se manifiestan también en su uso del suelo. Los aparcamientos cubren del 6 al $40 \%$ de las superficies urbanizadas, superficies que muchas veces son ineficaces, aumentando las distancias que los peatones deben caminar, incrementar los costes de construcción y dañando el medioambiente (Childs, 1999).

No hay duda sobre la gran contribución que el aparcamiento ha hecho a la ciudad difusa, fragmentando hábitats naturales, pero también hábitats humanos. Por ejemplo, los grandes aparcamientos son letales para anfibios y animales de pequeño tamaño además de disgregar notablemente el tejido urbano. En la ciudad compacta, sin embargo, el aparcamiento puede ser usado en horarios alternativos, al mismo tiempo que se logra reducir la superficie construida necesaria, ayudando a mantener una imagen más compacta de nuestro entorno urbano.

Aunque como hemos visto, el principal problema del uso del asfalto es su condición de impermeabilidad que crea graves dificultades en la gestión y evacuación del agua y su contribución a las islas de calor, también su aplicación contribuye a la generación de smog, hasta tal punto que en áreas altamente contaminadas se aconseja su puesta en obra durante los meses más fríos del invierno.

En los últimos años se han hecho grandes avances en el desarrollo de materiales permeables y resistente que se puedan usar para el tráfico rodado. Desde hormigones permeables (como el usado en BRIT por Balmori Associates) y asfaltos con aditivos reciclados que logran un resultado igual o mejor en cuanto a duración y mantenimiento, a pavimentos que ayudan a neutralizar el dióxido de carbono.

Si no es posible la utilización de materiales porosos en la totalidad del aparcamiento, siempre queda la posibilidad de emplear distintos materiales en correspondencia a las distintas partes y usos de los aparcamientos, sin olvidar la importancia de incluir áreas verdes permeables hacia las que dirigir las aguas de lluvia.

\section{Aparcamientos}

Todos estos indicadores medioambientales han empezado a ser considerados por los pocos diseñadores que han trabajado en el ámbito de los aparcamientos. El Botanical Reserch Institute of Texas es un claro ejemplo de estas nuevas técnicas de recolección y re-uso inteligente del agua de lluvia.

Desde la gran escala, BRIT es parte del corredor ecológico del río Trinity. La continuidad del paisaje es crítica para la salud de los grandes sistemas en los que se engloba, y el proyecto para BRIT debe ser observado desde este punto de vista. El emplazamiento no puede considerarse como un elemento aislado, sino como parte de una cuenca hidrográfica, lo que implica que el agua fluye en una dirección de acuerdo con su entorno. Todos los esfuerzos para contrarrestar este proceso solo pueden consumir mucho esfuerzo y dinero, a menudo sin resultados. También deberíamos considerar el paisaje en una escala mayor, casi aérea, para 


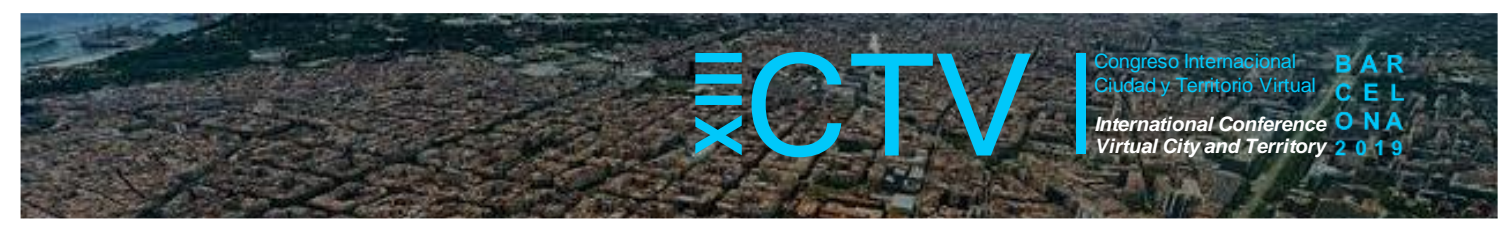

ver el entorno y descubrir las implicaciones de esta gran escala en la forma en que se vincula al lugar del proyecto. Todo lo que hacemos en un lugar se relaciona con su entorno y viceversa.

Al principio, el gran espacio que ocupaban las 358 plazas de estacionamiento requeridas, presenta un problema importante desde un punto de vista económico. Pero si consideramos el área de aparcamiento, más las cubiertas de los edificios como parte de un sistema activo de recolección de agua de lluvia y como una posible área de investigación, toda la intervención se convierte en un sistema operativo ecológico; ya no es un problema a resolver, sino un elemento para deleitar a los visitantes desde el momento en que estacionan sus automóviles. El agua recogida de los tejados puede acumularse en un tanque y reutilizarse para regar durante las estaciones secas.

Los materiales también siguen estas líneas de continuidad hidrológica. Las plazas de los automóviles están pavimentadas con asfalto poroso para facilitar la filtración del agua y no tienen separación con las áreas de vegetación. Solo existen pequeñas barreras para evitar que los coches invadan los jardines de lluvia. Las calzadas usan materiales impermeables, pero siempre vierten agua a las zonas permeables o directamente al parque.

Podemos distinguir cuatro áreas da plantación claramente diferentes: el área abierta detrás de los edificios se dedica a la Fort Worth Prairie; los edificios con muros vegetales y cubiertas ajardinadas donde se recoge el agua de lluvia; la zona de investigación donde hay una exhibición de hiervas nativas, y finalmente, el área de estacionamiento, donde los árboles existentes, los árboles nuevos y los rain gardens forman un continuo que articula todo el sitio.

Figura 3. Diagramas de gestión del agua. Botanical Research Institute of Texas de Balmori Associates (izquierda) / Herman Miller Factory de MVVA (derecha)

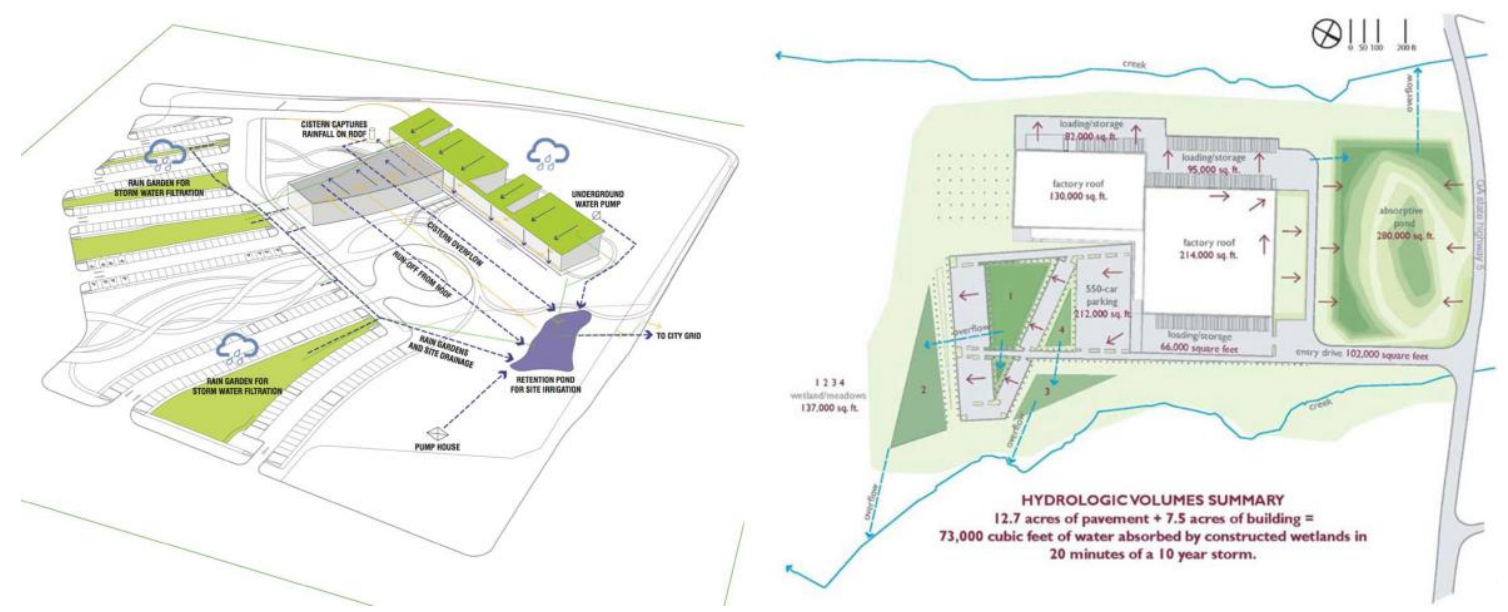

Fuente: Imagen cortesia de BalmoriAssociates (izquierda) y www.asla.org

Un sistema similar había sido previamente utilizado por Michael Van Valkenburgh en su proyecto titulado "12000 obreros cumplen con la ecología en el aparcamiento". La fábrica de producción y montaje de muebles Herman Miller está ubicada en un terreno de 70 acres en una zona rural de Georgia, donde el cliente quería un estacionamiento para 550 automóviles y 120 remolques dentro de un área total de 10 hectáreas. La escorrentía de las superficies de estacionamiento, las carreteras necesarias y los $330,000 \mathrm{~m} 2$ de cubiertas tendrían un impacto 


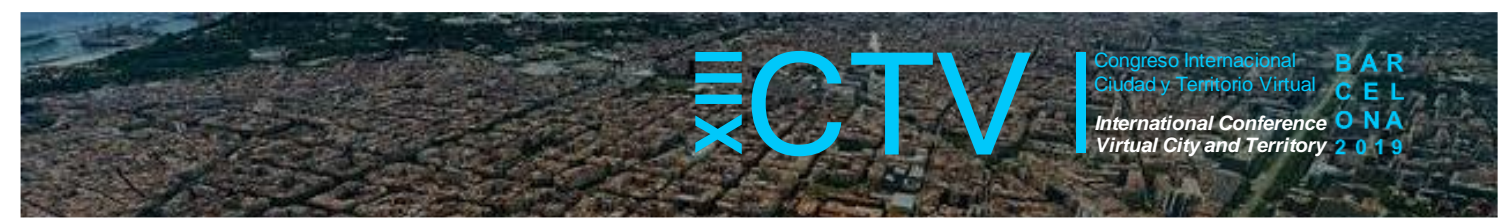

devastador en el frágil ecosistema circundante. Así, la primera acción tomada por MVVA fue establecer la captación del agua pluvial como prioridad esencial para el proyecto; posteriormente se consideró su tratamiento y su lenta liberación en el entorno para no crear una violenta aportación de agua en el ecosistema adyacente.

MVVA integra la ecología de forma elegante y honesta con enormes superficies pavimentadas, creando un nuevo modelo de paisaje respetuoso con el medio ambiente y de bajo mantenimiento. Este modelo se puede aplicar con igual éxito en áreas urbanas y suburbanas, y muestra cómo los arquitectos paisajistas pueden jugar un papel crucial en la producción de un sistema de gestión hidrológica eficaz a través de un buen diseño. Se entiende así el por qué la fábrica Herman Miller fue el único proyecto de estacionamiento desde 1990 que ganó el Premio ASLA Design Honor 2005 de la Sociedad Americana de Arquitectos Paisajistas.

En lugar de crear un amplio estacionamiento, fue diseñado como un mosaico de pequeños lotes triangulares sin bordillos, colocados en terrazas planas plantadas con vegetación pantanosa donde el agua se asentaría durante las tormentas y se filtraría lentamente a través del suelo, recordando los campos de arroz.

Para eliminar los bordillos, las tuberías y las bocas de inspección, el equipo MVVA colocó el edificio principal en un lugar predominante y moldeó todo el terreno con un gradiente del $5 \%$ para permitir que el agua de lluvia de toda el área llegue a las áreas permeables.

Este sistema hidrológico de bioingeniería es la base del diseño del proyecto paisajístico. El aparcamiento ha sido rediseñado como parte de un sistema ecológico más que como una barrera para los procesos ecológicos; secciones de los humedales actúan junto con las franjas de estacionamiento para purificar el agua, detener la escorrentía del agua de lluvia, prevenir la erosión y favorecer hábitats silvestres.

Un paso más lo dan Michel Desvigne y Christine Dalnoky en su proyecto para la Thomson Factory en Guyancourt (Francia) donde la vida útil de la fábrica y del aparcamiento asociada a la misma están en la base del proyecto de paisajismo. Se plantea todo un sistema de plantación y vegetación que establezca un sistema ordenado de vegetación alrededor de la edificación mientras ésta esté en funcionamiento y que posteriormente, cuando ésta quede obsoleta, pueda colonizar todo el espacio construido. Dichos sistemas vegetales forman asimismo la estructura de la zona, aún por definir cuándo se realizó este proyecto. Cabe destacar el detallado estudio realizado sobre la utilización de las distintas plazas de aparcamiento en función de su cercanía a la edificación, siendo las más cercanas a la misma las más construidas mientras las más alejadas que son menos utilizadas, se mantienen en un estadio más natural, que propicie la reconquista de su espacio con el finalizar de la actividad industrial.

\section{Conclusiones}

Por lo tanto, menos cemento y más territorio, más verde y espacios abiertos. Un punto esencial para cambiar el enfoque del diseño al centrarse en el desafío del cambio climático se refiere precisamente a los espacios abiertos, incluso los más pequeños, desde las calles hasta los intersticios urbanos o los jardines privados: este conjunto de espacios ya no puede ser considerado como "vacío" o como una simple red de conexión dentro de la ciudad, si no como 


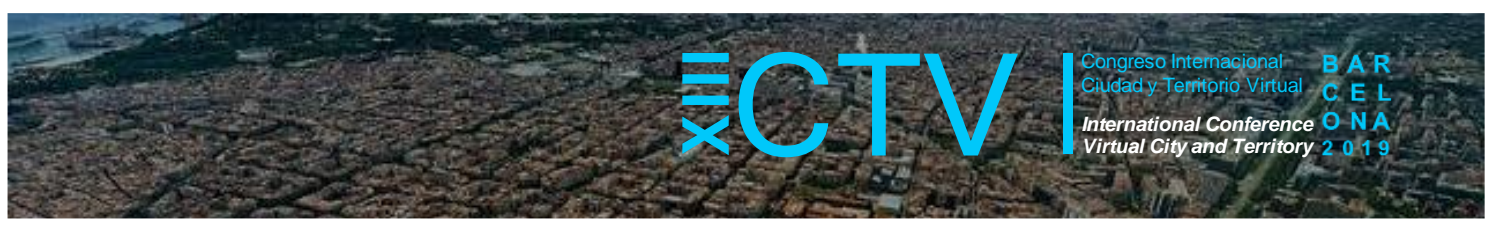

una densa trama verde y azul, que, por lo tanto, no configura lugares para cruzar distraídamente, sino lugares para ser proyectados como una oportunidad para que la ciudad pueda respirar y mitigar los efectos del cambio climático.

Diferentes son los enfoques y muy diferentes son los contextos ambientales y urbanos en los que se ubican los proyectos contemporáneos, pero cada uno de ellos participa de la necesidad de gestionar un clima que cambia cada vez más rápido. La respuesta del diseño no siempre es unívoca, pero va sin duda en la dirección marcada por las palabras de Diana Balmori: "Debemos poner la ciudad del siglo XXI en la naturaleza en lugar de poner la naturaleza en la ciudad" (Balmori, 2010)

Contribuciones de los autores: La primera autora ha desarrollado los apartados 1. El cambio climático y el proyecto de paisajismo, 2. Cambia el clima, cambia el proyecto de paisajismo, 3. Experiencias, estrategias y buenas prácticas de proyecto; la segunda autora ha desarrollado los apartados 4. Hacia una ciudad permeable, 5. Aparcamientos; Ambas autoras conjuntamente han desarrollado el resumen, las conclusiones y la bibliografía.

Conflicto de Intereses: Las autoras declaran que no hay conflicto de intereses.

\section{Bibliografía}

Andreani, F. (1995). Parcheggi. Storia, Opere, Idee. Gamgemi Editore

Balmori, D. and Benoit, G. (2007). Land and Natural Development (LAND) code. Guidelines for sustainable land development. Hoboken New Jersey: John Wiley \& Sons INC.

Balmori, D. (2010). A Landscape Manifesto. New Haven and London: Yale University Press

Bassolino, E. (2019). The impact of climatechange on local water management strategies. Learning from Rotterdam and Copenhagen. In UPLanD, n4, pp. 21-40.

Ben-Joseph, E. (2012). Rethinking a lot. The design and culture of parking. Massachusetts Institute of Technology

Calzolari, V., Álvarez Mora, A. (2012). Paesistica/Paisaje. Valladolid, España: Universidad de Valladolid.

Childs, M. (1999). Parking Spaces. A design, implementation and use manual for architects, planners and engineers. McGraw-Hill

City of Copenhagen (2011). CopenhagenClimate Adaptation Plan.

City of Rotterdam (2013). Rotterdam ClimateChange Adaptation Strategy.

City of Singapore (2017). Urban Systems Studies. The active, beautiful, cleanwatersprogramme: water as an environmental asset. Centre for LiveableCities.

Di Gregorio, R. (2019). Il progetto di paesaggio come risposta alle sfide climatiche. https://www.edilportale.com/news/2019/05/ambiente/il-progetto-di-paesaggio-come-rispostaalle-sfide-climatiche 70277 52.html 


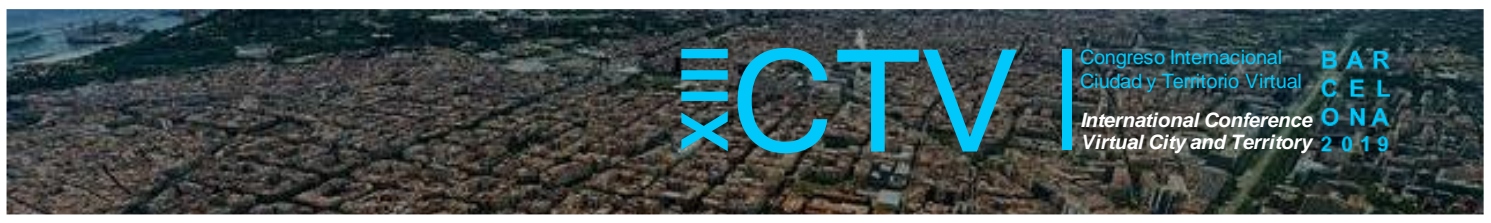

Di Paola, M. Pellegrino, G. (2018) Nell'Antropocene. Etica e politicaalla fine di un mondo. Roma: DeriveApprodi

Fioravanti, G., Rossi, P.O., Balbo, P.P., Cellini, F. (1999). Per un progetto urbano. Del governo della sosta ad una strategia per Roma. Fratelli Palombi Editori

Formenti, E. (2014). Big U, Rebuild by design, Lower Manhattan. In Arketipo, n 85/14, pp. $42-$ 55.

Groth, P. (1990). Parking Gardens in The Meanign of gardens. Idea, place and action. Edited by Mark Francis and Randolph T. Hester, JR. Cambridge, Massachussets. The MIT Press.

Jakle, J. A. and Sculle, K. A. (2004). Lots of parking. Land use in a car culture. University of Virginia Press, Charlottesville and London

Manigrasso, N. (2012). Verso la Città ad_Attiva. Rispondere ai cambiamenti climatici attraverso una nuova concezione del tempo nei processi e negli esiti progettuali. En: Atti della XV Conferenza Nazionale SIU - L'urbanistica che cambia. Rischi e Valori. Pescara, Italia.

Onipede, B. O. (2017). Combating climate change with landscape design. Bells University of Technology, Ota.

Mayer, M. R. (2005). Parking Lots: an investigation of public space in the Contemporary American City. Georgia Institute of Technology

Mattogno, C. (2014). "Ambiente" in Ventuno parole per l'urbanistica. Roma: ARACNE editrice.

Meijerink, P. (1998). Lestationnementgenerique. In Inter: art actuel, n69, 1998, p.34-35. Recuperado de http://id.erudit.org/iderudit/46316ac

Miller, C. G. (1988). Carscape: a parking handbook, Inwin-Sweeney-Miller Foundation

Paolinelli, G. (2018). Landscape Design in a ChangingWorld. Firenze, Italia: DidaPress.

Shoup, D. (2003). The truth in transportation Planning. Recuperado de http://shoup.bol.ucla.edu/TruthInTransportationPlanning.pdf

Shoup, D. (2011). The high cost of free parking. American Planning Association Planners Press, Chicago | Washington DC

Traldi, L. (2018). Design e ClimateChange. L'architettura del paesaggio che salva la vita.In Dcasa, novembre 2018, pp. 56-60.

Trancik, R. (1986). Findinglostspace. Theories of urban design. John Willey and Sons

Zardini, M. a cura di (1996). Paesaggi ibridi. Un viaggio nella città contemporanea.Skira

Zardini, M. a cura di (2003). Asfalto: il carattere della città. Electa 